\section{Datenbanken zu Kriegen und bewaffneten \\ Konflikten:}

\author{
Arbeitsgemeinschaft Kriegsursachenforschung (AKUF) der Uni- \\ versität Hamburg (seit 1945): http://www.akuf.de \\ Correlates of War (CoW) (1816-2007): http://www.correlatesofwar. \\ org
}

Escola de Cultura de Pao der Universität Barcelona (seit 2001): http://escolapau.uab.cat

Heidelberger Institut für Internationale Konfliktforschung e.V. (HIIK) (seit 1945): http://www.hiik.de

Uppsala Conflict Data Program (UCDP) der Universität Uppsala (seit 1945): http://www.pcr.uu.se

\title{
Für eine Stärkung des Parlamentsbeteiligungsgesetzes
}

\author{
Kommission „Europäische Sicherheit und Zukunft der Bundeswehr" am IFSH*
}

$\mathrm{D}$ as Grundgesetz verpflichtet die Bundesregierung, wie es im Urteil des Bundesverfassungsgerichts vom 12. Juli 1994 heißt, „für einen Einsatz bewaffneter Streitkräfte die grundsätzlich vorherige konstitutive Zustimmung des Deutschen Bundestages einzuholen." Das Urteil des Bundesverfassungsgerichts zum Lissabon-Vertrag vom 30. Juni 2009 stellt klar, dass der Parlamentsvorbehalt integrationsfest ist und zum unantastbaren Kern der grundgesetzlichen Verfassungsidentität gehört. Seit 2005 bestimmt das Parlamentsbeteiligungsgesetz (ParlBG) die Regeln und das Verfahren der konstitutiven Zustimmung des Bundestags zu bewaffneten Auslandseinsätzen.

Dem ehemaligen Verteidigungsminister Thomas de Maizière scheinen die Mitwirkungsrechte schon seit Langem offenbar zu engmaschig gestrickt. Insbesondere befürchtet er Konflikte zwischen der Ausweitung gemeinsamer militärischer Strukturen und Fähigkeiten in NATO wie EU einerseits und den im Gesetz festgeschriebenen Beteiligungsrechten des Parlaments andererseits. Bereits in den Verteidigungspolitischen Richtlinien von 2011 heißt es: „Ob und inwieweit die Zusammenarbeit in Bündnissen und die sich wandelnde Sicherheits- und Bedrohungslage rechtlichen Anpassungsbedarf nach sich ziehen, wird zu analysieren sein." In einem Interview in der ZEIT vom 16. Mai 2012 bekennt er sich ausschließlich zum „Rückholrecht“ bzw. zu einem „Vetorecht" des deutschen Parlaments. In der vom Verteidigungsminister erlassenen „Konzeption der Bundeswehr" vom Juli 2013 heißt es: „Neben der politischen Bedeutung hat die multinationale Zusammenarbeit erhebliche militärische und praktische Auswirkungen. Sie kann Handlungsoptionen erweitern, die Durchhaltefähigkeit eigener Kräfte erhöhen und Kosten reduzieren; umgekehrt ergeben sich aus Bündnisverpflichtungen planerische Vorgaben an die eigenen Fähigkeiten. Deshalb bedarf die multinationale Zusammenarbeit grundsätzlich der kontinuierlichen politischen Abstimmung und kann das Zurückstellen nationaler Vorbehalte erfordern."

\footnotetext{
* Folgende Mitglieder der Kommission haben die Stellungnahme erarbeitet bzw. tragen sie mit: Detlef Bald, Jörg Barandat, Michael Brzoska, Hans-Georg Ehrhart, Hans-Günter Fröhling, Jana Hertwig, Sabine Jaberg, Berthold Meyer, Reinhard Mutz, Winfried Nachtwei, Bernhard Rinke, Jürgen Rose, Claus von Rosen, Paul Schäfer, Michael Staack.
}

Das eröffnet Raum für Spekulationen, aber auch für politische Initiativen. So plädieren Andreas Schockenhoff und Roderich Kiesewetter von der CDU für einen parlamentarischen Vorratsbeschluss - also die Erteilung einer Generalvollmacht für die Regierung für den Einsatz der Bundeswehr -, um auf dieser Basis der Exekutive das „Einsatzrecht“ und der Legislative das „Rückholrecht“ zuzuweisen. Auch die Wissenschaft beteiligt sich mit Vorschlägen zur Ergänzung des ParlBG. Nach einem Vorschlag aus der Stiftung Wissenschaft und Politik sollte die Zustimmungspflicht für die Teilnahme deutscher Soldatinnen und Soldaten an Einsätzen zwei Ausnahmen umfassen: Die bisherige Praxis deutscher Mitwirkung an den Hauptquartieren der integrierten Kommandostruktur sollte beibehalten und der Entwicklung zu mehr Flexibilität angepasst werden. Darüber hinaus sollte die Teilnahme an der bündnisgemeinsamen Aufklärungs- und Führungsfähigkeit sichergestellt werden.

Eine andere Diskussionslinie zielt auf eine Schließung von Lücken im ParlBG. Bedarf für eine Stärkung gibt es beispielsweise in der Frage eines Einsatzes von Spezialkräften. Neue technologische Möglichkeiten der Fernwirkung, wie ein Einsatz bewaffneter Drohnen, auch vom Boden der Bundesrepublik aus, oder im Rahmen eines „Cyberwar“, könnten zu Lücken in der Parlamentsbeteiligung führen, die es präventiv zu schließen gilt.

Die jüngeren Diskussionen um das ParlBG berühren aber nicht nur aktuelle Fragen der internationalen Integration der Bundeswehr und andere Grenzfälle der Parlamentshoheit über die Bundeswehr. Im Kern geht es um den Stellenwert, welcher der parlamentarischen Mitwirkung beim Auslandseinsatz generell zukommt.

- Entwickelt sie sich zum Ballast für die deutsche Sicherheitspolitik?

- Torpediert der Bundestag mit seinen Verfahren und Entscheidungsbefugnissen militärische Kooperation im Bündnis oder der EU, zumal diese eingedenk knapper Kassen arbeitsteiliger werden dürfte?

- Soll die Parlamentsbeteiligung beim bewaffneten Auslandseinsatz als das institutionelle Rückgrat einer Kultur der Zurückhaltung gestutzt werden, weil auf Deutschland künftig mehr militärische Verantwortung zukommt? 
In den Koalitionsverhandlungen zwischen CDU, CSU und SPD ist vereinbart worden, dass eine Kommission binnen Jahresfrist prüft, wie auf dem Weg fortschreitender Bündnisintegration und trotz Diversifizierung der Aufgaben die Parlamentsrechte gesichert werden können. Im Folgenden nehmen wir zur Diskussion über die Fortentwicklung der parlamentarischen Kontrolle der Streitkräfte Stellung. Nach einer Betrachtung der von verschiedenen Seiten vertretenen Argumente plädieren wir für eine Stärkung des Parlamentsbeteiligungsgesetzes. Aus unserer Sicht ist aktuell vor allem die Kontrolle von Spezialkräften problematisch.

\section{Argumente für eine Einengung der parlamentarischen Beteiligung}

Einfluss: Um seine nationalen und europäischen Interessen in einer globalisierten Welt vertreten zu können, muss Deutschland als „Gestaltungsmacht" agieren. Dazu bedarf es einer starken und handlungsfähigen Exekutive, zu deren Instrumenten angemessene militärische Mittel gehören. Über den Einsatz von Streitkräften muss die Regierung ggf. rasch im deutschen Interesse entscheiden können. „Der Preis von Einfluss ist Verantwortung“, so der ehemalige Verteidigungsminister Thomas de Maizière. Die Rechenschaftspflicht gegenüber dem Bundestag bleibt ebenso bestehen wie dessen Rückholrecht.

Bündnispolitik: Deutschland setzt seine Streitkräfte grundsätzlich nur im Rahmen von Organisationen kollektiver Sicherheit und Verteidigung ein (Vereinte Nationen, NATO, EU). Die Bündniszugehörigkeit gehört gewissermaßen zur Staatsräson. Dafür sind historische Erfahrungen und sicherheitspolitische Erwägungen verantwortlich. Die Bundeswehr ist Parlaments- und Bündnisarmee gleichermaßen. Die NATO bindet die für die deutsche und europäische Sicherheit unverzichtbaren nordamerikanischen Partner an Europa. Darum stellt Bündnissolidarität gerade in der Frage des bewaffneten Einsatzes von Streitkräften ein hohes Gut dar, das Deutschland pflegen muss. Das ParlBG erschwert hingegen eine tiefergehende militärpolitische Integration und führt bei den Bündnispartnern zu Zweifeln an der Verlässlichkeit Deutschlands.

Einsatzfähigkeit: Knappe Mittel, der Druck zur Anpassung der Streitkräftestrukturen und zur Modernisierung der militärischen Fähigkeiten führen zu verstärkter multilateraler Zusammenarbeit in Form des Zusammenlegens von Kapazitäten und der Rollenspezialisierung. Dadurch sollen Effizienz und Effektivität des Bündnisses gestärkt werden. Dieses Vorgehen setzt Verlässlichkeit der Einsatzfähigkeit voraus. Nationale Vorbehalte und Sonderverfahren lassen sich damit schlecht vereinbaren. Darum bedarf es einer Vorabzustimmung („Vorratsbeschluss“) des Bundestages zu Einsatzkonzepten integrierter Verbände und einer Regelung, welche die Mitwirkung deutscher Soldatinnen und Soldaten in bündnisgemeinsamen Führungs- und Aufklärungsfähigkeiten jederzeit gewährleistet.

\section{Argumente für eine Stärkung der Rolle des Parlaments}

Gegen eine Schwächung des Wirkungskreises des Parlamentsbeteiligungsgesetzes werden die folgenden Argumente angeführt:
Friedenspolitik: Das Parlament wacht darüber, ob und wann Deutschland militärische Gewalt anwendet. Der Handlungsspielraum für die legitime Gewaltanwendung ist sowohl vom Völkerrecht als auch vom Grundgesetz bewusst sehr eng gefasst. Nach dem geltenden Völkerrecht muss entweder ein bewaffneter Angriff auf einen Staat vorliegen oder unmittelbar bevorstehen. Der betroffene Staat darf sich dann selbst - auch gemeinsam mit anderen Staaten - in Ausübung des in Artikel 51 UN-Charta anerkannten Rechts der individuellen oder kollektiven Selbstverteidigung gegen den Aggressor wehren. Oder aber der Sicherheitsrat der Vereinten Nationen muss ein entsprechendes Mandat nach Kapitel VII UN-Charta erteilt haben. Dann sind auch militärische Maßnahmen im Dienste des Weltfriedens und der internationalen Sicherheit erlaubt. Das Urteil des Bundesverfassungsgerichts von 1994 legt fest: Der Rückgriff auf militärische Mittel verlangt ein entsprechendes Mandat des Bundestages. Im AWACS/Türkei-Urteil vom Mai 2008 konkretisierte das Gericht: „Angesichts der Funktion und Bedeutung des wehrverfassungsrechtlichen Parlamentsvorbehalts darf seine Reichweite nicht restriktiv bestimmt werden. Vielmehr ist der Parlamentsvorbehalt (...) im Zweifel parlamentsfreundlich auszulegen." Politisch betrachtet kann nur eine starke Legislative gewährleisten, dass einerseits der parlamentarische Rückhalt einen Bundeswehreinsatz ausreichend legitimiert und damit auch die Chance für eine angemessene Unterstützung in der Bevölkerung besteht. Andererseits reduzieren Transparenz und kritische Debatte die Gefahr eines leichtfertigen Einsatzes.

Bündnispolitik: Bündnissolidarität ist ein hohes Gut. Verstärkte Integration kann Kosten sparen und Kräfte bündeln. Internationale militärische Zusammenarbeit basiert aber nicht auf Vasallentreue, sondern auf grundlegenden gemeinsamen Sicherheitsinteressen und Werten. Diese erlauben eine angemessene Reaktion, deren konkrete Ausgestaltung gerade im Bereich des Krisenmanagements den einzelnen Bündnismitgliedern obliegt. Eine automatische militärische Beistandsverpflichtung sieht der NATO-Vertrag nicht einmal für den Fall eines bewaffneten Angriffs auf einen Partner vor. Der Vertrag von Lissabon enthält zwar eine solche Bündnispflicht (Art. 42 Abs. 7 EU-Vertrag), lässt aber gleichzeitig ausdrücklich den „besonderen Charakter" der Verteidigungspolitik einzelner Mitgliedstaaten unberührt. Umso weniger kann eine automatische Verpflichtung zu gemeinsamem Handeln in Situationen gelten, die keine unmittelbare Bedrohung darstellen. Dementsprechend engagierten sich Bündnismitglieder in Afghanistan, im Kosovo und in Libyen in unterschiedlicher Weise und teilweise überhaupt nicht. Bündnissolidarität findet in der Frage des bewaffneten Einsatzes von Streitkräften in der Krisenbewältigung manchmal ihre Grenzen in politischen Erwägungen, ohne dass dadurch die Verlässlichkeit des Partners ernsthaft in Frage steht. Anderslautende Behauptungen sind eher taktischer Natur, um den politischen Druck zum Abbau der Parlamentsbeteiligung zu erhöhen.

Einsatzfähigkeit: Eine Vorabzustimmung des Parlaments zu Einsätzen integrierter Verbände, Stäbe und Hauptquartiere würde das verfassungsrechtlich garantierte Recht des Bundestages, über den militärischen Einsatz der Bundeswehr zu entscheiden, in unakzeptabler Weise einschränken. Grundgesetzliche Pflichten und Rechte stehen über Effektivitätsüberlegungen. Zudem 
wäre es gerade aus Sicht der NATO hoch problematisch, die Einsatzfähigkeit des Bündnisses von der zwingenden Teilnahme aller Mitgliedstaaten abhängig zu machen. Gemeinsame Strukturen müssen auch dann funktionsfähig bleiben, wenn einzelne Mitglieder sich nicht zu einer Beteiligung entschließen, was im Übrigen genauso durch Regierungen zu erwarten ist wie durch Parlamente. In gemeinsamen Strukturen muss daher, solange Staaten souverän entscheiden, ein gewisses $\mathrm{Maß}$ an redundanten Kapazitäten vorgehalten werden, das dieses Souveränitätsrecht abbildet. Dies ist gängige Praxis in der NATO, weil die Erfahrung gravierender Krisensituationen, wie etwa über den Irakkrieg 2003, gezeigt hat, dass sich nationale Interessen nicht durch integrierte Strukturen aushebeln lassen. Ist Gefahr im Verzug, ermöglicht das ParlBG bereits heute eine nachträgliche Zustimmung des Parlaments.

\section{Bewertung}

Die Argumente gegen eine Einschränkung der Zustimmungspflicht des Bundestages überzeugen vor allem aus zwei Gründen mehr als die Argumente der Befürworter:

Gelebte Praxis: Es ist empirisch belegt und in der öffentlichen Debatte Konsens, dass das ParlBG Einsätze bislang nicht behindert hat. Das räumen sogar seine Kritiker ein. Immerhin hat das Parlament bislang immer, wenn die Bundesregierung einen Antrag auf Entsendung der Bundeswehr oder Fortsetzung des Einsatzes gestellt hat, seine Zustimmung erteilt - in insgesamt über 70 Fällen seit 2005. Allenfalls äußert sich gelegentlich der kontrafaktische Verdacht, dass strittige Fälle - wie z.B. eine Ausweitung des deutschen ISAF-Einsatzes in Afghanistan nach Süden - wegen möglicherweise mangelnder Zustimmung im Parlament gar nicht erst zur Abstimmung gelangt seien. Der empirische Befund zum ParlBG ist über die politische Kontroverse hinweg einfach festzustellen: Es hat bislang weder Einsätze verhindert noch die Exekutive geschwächt.

Verhältnis Regierung/Parlament: Die Entkoppelung der Teilnahme an integrierten Strukturen, die bewaffnete Einsätze führen (z.B. Einsatzstäbe) und umsetzen (z.B. AWACS, Allied Ground Surveillance), von der Entsendeentscheidung birgt das Risiko, dass der Parlamentsvorbehalt ausgehöhlt wird. Die Nutzung bündnisgemeinsamer Einrichtungen beim Krisenmanagement steht auch ohne die Begrenzung der $\mathrm{Zu}$ stimmungspflicht des Bundestages unter dem Vorbehalt der Zustimmung der Mitgliedstaaten. Eine Änderung der Zustimmungsrechte würde also vermutlich nur eine Verschiebung des Gewichts von Regierung und Parlament bedeuten, ohne dass eine höhere Verlässlichkeit einer deutschen Beteiligung erzielt würde. Mithin geht dieses Argument der Kritiker ins Leere. Unbedingte Verlässlichkeit ließe sich nur erzielen, wenn die Entscheidungsbefugnis auf internationale Institutionen oder zwischenstaatliche exekutive Gremien überginge. Dies wäre aber mit dem Konzept eines Parlamentsheeres wie der Bundeswehr nicht mehr vereinbar. Sollten hier neue Optionen ermöglicht werden, die zumindest diskussionswürdig sind, dann müssten zuerst ergänzend zu den nationalstaatlichen Verfahren demokratisch legitimierte, überstaatliche parlamentarische Strukturen entstanden sein.

\section{Problemfall Spezialkräfte}

Das Parlamentsbeteiligungsgesetz von 2005 geht nicht gesondert auf den Aspekt der Einsätze von Spezialeinheiten ein. In Mandaten für Einsätze der Bundeswehr ist auch die Beteiligung von Spezialkräften enthalten gewesen. Die Kontrolle der Aktivitäten durch die Bundestagsabgeordneten ist allerdings begrenzt. Das Kommando Spezialkräfte (KSK) wird durch die Bundeswehrführung eingesetzt. Das Bundesministerium der Verteidigung hat in der Vergangenheit die Obleute der Bundestagsfraktionen des Verteidigungs- und des Auswärtigen Ausschusses vor Entsendung von Spezialkräften und während der Einsätze im Abstand von sechs Monaten (unter bestimmten Voraussetzungen auch nach wichtigen Einzeloperationen laufender Einsätze) unterrichtet. Dies geschah auf freiwilliger Basis. Damit lässt sich zweifellos von Parlamentsbeteiligung sprechen; zumal mit Blick auf Afghanistan gleich zwei Bundestagsmandate vorlagen - eines für die Operation Enduring Freedom (OEF), eines anderes für die UN-mandatierte Mission ISAF. Die bisherige Praxis der Geheimhaltung hat aber dennoch gezeigt, dass diese in Widerspruch zum Anspruch des Parlaments auf eine detaillierte Unterrichtung geraten kann. In den Koalitionsverhandlungen von CDU, CSU und SPD ist vereinbart worden, die „bewährte Form“ der Unterrichtung des Bundestages fortzusetzen. Es drängt sich allerdings die Frage auf, ob es für die parlamentarische Kontrolle ausreicht, wenn lediglich 14 Bundestagsabgeordnete über solche Einsätze informiert werden und die Obleute zudem zur Verschwiegenheit gegenüber ihrer Fraktion verpflichtet sind. Da bisher für das KSK keine besondere gesetzliche Grundlage geschaffen wurde, wie etwa mit dem BNDGesetz für den Bundesnachrichtendienst geschehen, sollten Einsätze von KSK-Soldaten, was die parlamentarische Kontrolle und insbesondere die Unterrichtungspflicht des Bundestages angeht, grundsätzlich nicht anders behandelt werden als andere Einsätze.

\section{Schlussfolgerungen}

Das ParlBG stellt keinen Ballast für eine effektive Sicherheitspolitik dar. Es verhindert auch nicht per se eine stärkere militärische Integration im Bündnis oder in der EU. Es stellt vielmehr eine höchstrichterlich bekräftigte Forderung an unser demokratisches Gemeinwesen dar, wenn es um die schwerwiegende Frage des Gewaltmitteleinsatzes geht. Dies gilt insbesondere dann, wenn es sich um solche Situationen handelt, die nicht durch das Recht auf Selbstverteidigung gedeckt sind. Im Idealfall verhindert die Parlamentsbeteiligung übereilte Entscheidungen, ermöglicht öffentliche Kontrolle, erhöht die Legitimität des Einsatzes und stärkt die Sicherheit Deutschlands. Um diesem Ideal näherzukommen, sollte der Bundestag das ParlBG um eine Regelung für den Einsatz von Spezialkräften ergänzen. Lücken der Parlamentsbeteiligung, die sich durch neue technologische Fähigkeiten ergeben könnten, sollten präventiv geschlossen werden.

Wir begrüßen die Einrichtung einer Kommission, wie in den Koalitionsverhandlungen von CDU, CSU und SPD vorgesehen, die den Auftrag hat, die Parlamentsrechte unter sich verändernden Bedingungen sicherzustellen. Sollte es zu einer Einsetzung einer solchen Kommission kommen, kann ihr Ziel nicht die Schwächung, sondern muss die Stärkung des Parlamentsbeteiligungsgesetzes sein. 